in a short time if chaos is to be prevented. Many London clinicians and managers are disgruntled about the delay in capital programmes and the granting of trust status that this latest inquiry has brought. But Mr Waldegrave's move to go beyond the market and develop a strategic London wide approach should be greeted with relief.

Properly executed, Sir Bernard's work should mean an end to the miasma of uncertainty and depression that has clouded London's health services since the NHS reforms were announced. In time, it should even provide answers to the questions "Which teaching hospitals should close?" and "Will there be one regional health authority for London?"

Director,

VIRGINIA BEARDSHAW

King's Fund London Initiative,

2 Palace Court,

London W2 4HS

1 Rivett G. The development of the London hospital system 1823-1982. London: King's Fund, 1986.

3 College of Health. Guide to hospital waiting lists 1991 . London: College of Health, 1991.

\title{
Clinical trials in general practice
}

\section{Learning from the failures}

In this issue Tognoni et al describe their attempt to evaluate the role of treatment of the elderly with isolated systolic hypertension in a general practice setting ( $\mathrm{p} 000) .{ }^{1}$ They had already conducted a successful collaborative prospective study in this setting, ${ }^{2}$ but on this occasion they failed.

They should, however, be congratulated on their honest and clear presentation of the problems that arose in their second study - predictable, although often glossed over in accounts of other trials in general practice. Their main problem was the large discrepancy between the number of general practitioners who agreed to participate and the few who actually started recruiting patients. This was despite the study's protocol being produced after close consultation with the general practitioners. The authors' discussion of their difficulties should benefit others proposing to embark on drug trials in general practice. Although difficult, such trials are essential: recommendations for treatment and management within general practice are useful only if actually feasible in the standard good general practice setting.

Recognising and treating hypertension are appropriate tasks for general practice. Previously, treatment of the few patients with malignant hypertension was begun in hospital and continued in outpatient clinics. But now we are faced with many more patients with milder hypertension, discovered as the result of initiatives in general practice, who are considered to merit treatment. The logistics of this undertaking require that treatment must be based mainly in general practice.

Several accounts of the design, conduct, and findings of trials for treating hypertension in general practice, or in the international setting most appropriate for comparison, have been published. ${ }^{3-6}$ Much can go wrong. The recruitment, selection, equipping, training, motivation, and quality control of the doctors, nurses, and clerks participating in the research; the design and operation of the central survey unit responsible for the day to day handling of incoming data; the provision of supplies; and the running analyses of the data are demanding of time, energy, and resources. To this must be added the contributions of working parties, ethics committees, and monitoring committees. Though the costs of properly conducted studies in general practice are high, the benefits of improved health and the savings that follow abandoning ineffective or hazardous treatment and introduc- ing a useful new treatment may far outweigh them. Because general practice varies among countries, extrapolating unreservedly from a trial in one country to another may not be possible: some duplication is unavoidable.

Once the research mechanism is in place it can be exploited by further studies. This policy was adopted by the Medical Research Council, which, having successfully completed a study of mild hypertension in general practice, ${ }^{6}$ continued to take full advantage of the established framework with a series of other studies.

If one were planning a survey of any size in general practice one would begin by going to published accounts of similar studies. These would suggest potential problems and how they might be overcome. Unfortunately, however, when the surviving author of a long term study gets down to writing an account of a decade of endeavour constraints of space alone preclude the presentation of all the details that would be useful to successors, and familiarity may take for granted hard won knowledge.

Tognoni et al are unlikely to create a fashion for the writing up of failed studies and their submission to journals-even if editors were prepared to publish them. Yet factors contributing to the failure as well as to the success of clinical trials in general practice merit periodic review. Perhaps these authors' frankness will persuade other scientists to follow suit, to add accounts of their important failures to the literature. Past failures could contribute to future successes.

GILLIAN GREENBERG

74 North End Road,

London NW11 7SY

\footnotetext{
Tognoni G, Alli C, Avanzini F, Bettelli G, Colombo F, Corso R, et al. Randomised clinical trials in general practice. BMf 1991;303:969-71.

2 Avanzini F, Alli C, Bettelli G, Colombo F, Conforti L, Pirone F, et al. Feasibility of a large prospective study in general practice: an Italian experience. $B M \mathcal{F}$ 1987;294:157-60.

3 Hypertension Detection and Follow-up Program Cooperative Group. Five year findings of the hypertension detection and follow-up program. (1) Reduction in mortality of persons with high blood pressure, including mild hypertension. (2) Mortality by race, sex, and age. JAMA 1979;242:2562-77.

4 Australian National Blood Pressure Study Management Committee. The Australian therapeutic trial in mild hypertension. Lancet 1980;i:261-7.

IPPPSH Collaborative Group. Cardiovascular risk and risk factors in a randomised trial of treatment based on a beta-blocker oxyprenolol: the international prospective primary prevention study in hypertension. $\mathcal{F}$ Hypertens 1985;3:379-92.

6 Miall WE, Greenberg G. Mild hypertension: is there pressure to treat? Cambridge: Cambridge University Press, 1987.
} 